apparatus used for both low-energy and high-energy precision determinations of energy-levels, using for the latter the new Van de Graaff generator at the Massachusetts Institute. Results have been obtained for a variety of elements, in many places checking with those obtained elsewhere at low energy, and adding many new ones at high energy. Dr. Bender described the unique high-resolution beam analysing sxstem used with the University of Pittsburgh cyclotron. He then summarized the results so far obtained by its use. These consist of energy-levels obtained from $\left(p p^{\prime}\right),(p, \alpha)$ reactions ; many old levels have been found, some with new precision, and many new highly excited levels have been located. Finally, Dr. Way reported a new set of values for the binding energies of the nuclei above lead, and also in the region around neutron number 50 and atomic number 50. These were obtained by considering closed cycles involving alpha-decay and beta-decay. On the basis of the existing experimental data, differences in binding energies at both the neutron and proton 'magic' numbers showed up clearly.

Discussion leaders for the sessions included Dr. Maria Mayer (Argonne National Laboratory), Dr. Maurice Goldhaber (Brookhaven National Laboratory), Dr. S. T. Butler (Cornell), Dr. G. C. Wick (Carnegie Institute of Technology), and Dr. T. Lauritsen (California Institute of Technology).

Philip M. Steyce

\section{ISOTOPES IN MEDICINE}

$\mathrm{T}$ HE application of isotopes in medicine is a subject which shows signs of dangerous overpopularization, with the consequent unjustified simplifications of the problem. A recent publication, entitled "Isotopes in Medicine" *, must therefore be very welcome since this series of articles, a number of them reviews, by acknowledged authorities in their respective fields demonstrates clearly the limitations as well as the possibilities of the clinical and biochemical applications of isotopes. The title "Isotopes in Medicine" is misleading, since only a few of the direct applications of isotopes in medicine are discussed and a number of papers are devoted to reviews of biochemical investigations which are unlikely to exert much influence on clinical medicine for some time. This, however, is not meant as a criticism of the standard of the material, which is high, and the editor is to be congratulated on his choice.

Isotopes represent an important new weapon in the armoury of the clinician and the biochemist; but it is clear that they rarely provide a short cut to the solution of a given problem, and their use demands not only considerable technical resources but, above all, a wide knowledge and experience of the particular field in which they are to be applied. The use of radioactive isotopes in radiotherapy can only be based on a wide experience of orthodox methods of radiation therapy, as is clear from the contribution to this volume by Ralston Paterson and his colleagues. In biochemical research the use of isotopes has in a number of fields raised rather than solved problems, and advances in the applications of isotopes will, to a very large extent, depend on advances in general biochemical theory and technique.

The basic problem in the clinical application of radioactive isotopes is that of obtaining adequate localization of the isotope within the required tissue.

*Brit. Med. Bull., 8, Nos. 2-3, 111-300 (1952), 188.
A degree of localization is required for both therapeutic and diagnostic applications, but in therapeutic work a much higher degree of localization is needed in order that the healthy tissues of the body should not suffer too great damage. The concentration of iodine by the thyroid has been made the basis of many diagnostic and therapeutic studies, which are well reviewed in this volume; but it must be admitted that the popularity of thyroid studies with iodine is largely due to the fact that iodine alone of all the elements will achieve by metabolic processes a high degree of localization within the body.

Increasing use is being made in radiotherapy of radioactive isotopes in the form of discrete sources used either externally or within the body. No papers on this aspect of the subject are included, which is perhaps reasonable since the problems are basically technical ones, and the clinical applications follow along the lines of treatment with X-rays and with radium sources. Other methods of providing a localized irradiation of the required tissues are being developed, and two of the recent techniques are discussed. A description is given of the treatment of malignant bladder conditions by a radioactive solution enclosed in a rubber balloon in the bladder, and of irradiation of the pleural and peritoneal cavities by the injection into these cavities of radioactive gold in colloidal form.

The diagnostic application of isotopes involves to a large extent radiation measurements made externally to the patient and thus must depend basically on instrumentation. An application is described of scintillation counting in an attempt to diagnose brain tumours following administration to the patient of fluorescein labelled with iodine-131. So far, in Great Britain, the results with this technique have not been very promising.

One of the most simple and direct applications of radioactive isotopes in diagnosis lies in the study of blood flow. Here a measure is required only of the rate of transport of radioactive material injected into the blood, and valuable clinical applications have been found for this technique, described in several papers of this volume.

On the technical side the volume contains a number of extremely useful articles, in particular that by L. H. Gray entitled "General Principles of Assay and Standardisation of Radioactive Isotopes". A report is also given by members of the staff of the Radiochemical Centre, Amersham, on the availability of labelled compounds, with details of the methods of synthesis of a number of compounds labelled with carbon-14.

L. F. LAMERTON

\section{NATIONAL INSTITUTE OF AGRICULTURAL ENGINEERING OPEN DAYS}

$T$ HE National Institute of Agricultural Engineering, at Wrest Park, Silsoe, Beds, held open days during July 22-24, when exhibits were staged to illustrate to visitors the work of the Institute. The exhibits did not cover the whole programme of the work in hand at Wrest Park (which is reported more fully in the recent report for $1949-51^{1}$ ) ; but, even so, they were quite comprehensive and representative of many aspects of the activities of the departments. 